\title{
WILEY-VCH
}

DOI: 10.1002/ ((please add manuscript number))

Article type: Communication

\section{Infrared organic light-emitting diodes with carbon nanotube emitters}

Arko Graf, Caroline Murawski, Yuriy Zakharko, Jana Zaumseil* and Malte C. Gather*

A. Graf, Dr. Y. Zakharko, Prof. J. Zaumseil

Institute for Physical Chemistry, Universität Heidelberg, D-69120 Heidelberg, Germany

E-mail: zaumseil@uni-heidelberg.de

A. Graf, Dr. C. Murawski, Prof. M. C. Gather

Organic Semiconductor Centre, SUPA, School of Physics and Astronomy,

University of St Andrews, St Andrews KY16 9SS, United Kingdom

E-mail: $\underline{\operatorname{mcg} 6 @ \text { st-andrews.ac.uk }}$

Keywords: OLED, single-walled carbon nanotube, near infrared, trion, emitter orientation

\begin{abstract}
While organic light-emitting diodes (OLEDs) covering all colors of the visible spectrum have been demonstrated, suitable organic emitter materials in the near-infrared (nIR) beyond $800 \mathrm{~nm}$ are still lacking. Here, we demonstrate the first OLED based on single-walled carbon nanotubes (SWCNTs) as the emitter. By using a multi-layer stacked architecture with matching charge blocking and charge transport layers, we achieve narrow band electroluminescence at wavelengths between $1000 \mathrm{~nm}$ and $1200 \mathrm{~nm}$, with spectral features characteristic of excitonic and trionic emission of the (6,5) SWCNTs used. We investigate the OLED performance in detail and find that local conduction hot-spots lead to pronounced trion emission. Analysis of the emissive dipole orientation shows a strong horizontal alignment of the SWCNTs with an average inclination angle of $12.9^{\circ}$ with respect to the plane, leading to an exceptionally high outcoupling efficiency of $49 \%$. Our SWCNT-based OLEDs represent a highly attractive platform for emission across the entire nIR.
\end{abstract}




\section{WILEY-VCH}

Due to the exceptional chemical tunability of organic materials, efficient OLEDs covering the entire visible part of the spectrum and even the very near-infrared, up to $800 \mathrm{~nm},{ }^{[1,2]}$ have been demonstrated. While current OLED technology has successfully entered the display and lighting industry, ${ }^{[3,4]}$ OLEDs emitting further in the nIR - at wavelengths beyond $800 \mathrm{~nm}-$ would facilitate a range of new applications, in particular in bio-imaging and skin treatment as well as in optical data communication and night-vision devices. However, very few organic materials emit in this spectral range and the ones that do typically suffer from poor emission efficiency. ${ }^{[5]}$ Previous work focused on either small molecules, ${ }^{[6-9]}$ polymers, ${ }^{[10]}$ polymer nanocrystals ${ }^{[11]}$ or solution processed quantum dots ${ }^{[12]}$. While these approaches certainly hold promise, important challenges remain. Some nIR emitting devices contain heavy metals, show undesirable emission in the visible or are not suitable for fabrication on flexible substrates. To overcome these limitations, we propose to use semiconducting single-walled carbon nanotubes (SWCNTs) as nIR emitters. ${ }^{[13]}$

SWCNTs exhibit intriguing optoelectronic properties, such as exceptional photostability and a diameter-dependent exciton energy, which gives rise to wide tunability of the emission wavelength. ${ }^{[14]}$ In addition, they offer excellent mechanical flexibility and compatibility with solution processing. Polymer-sorted SWCNTs have been used as strong nIR absorbers for efficiency enhancement in solar cells by harvesting. ${ }^{[15,16]}$ Recently, SWCNTs have also been employed to electrically generate light, ranging from single-photon emission $^{[17]}$ to exciton-polaritons in light-emitting field-effect transistors at extreme current densities. ${ }^{[18]}$ In OLEDs, however, SWCNTs have so far only been used as transparent electrodes $^{[19-23]}$ or as charge transport and charge blocking layers ${ }^{[24-26]}$.

Here, we demonstrate nIR-emitting OLEDs based on solution-processed semiconducting SWCNTs as the active emitter material, combined with matching chargeblocking materials and doped charge transport layers. For an optimized stack architecture, the 


\section{WILEY-VCH}

emission is exclusively in the $\mathrm{nIR}(>1000 \mathrm{~nm})$ and shows a very narrow linewidth $(<50 \mathrm{~nm}$ full width at half maximum (FWHM)). The one-dimensional nature of the nanotubes leads to nearly complete horizontal orientation of the transition dipole moments, resulting in a $60 \%$ increase of light outcoupling efficiency relative to an isotropically oriented emitter. The devices reach an external quantum efficiency (EQE) of $0.014 \%$, which in the future may be improved by modifications to the nanotubes. Interestingly, the electroluminescence (EL) is not always exclusively excitonic but instead local inhomogeneities can also lead to further red-shifted trion emission.

In a first step, monochiral $(6,5)$ SWCNTs were purified by selective polymerwrapping with PFO-BPy using a high-speed shear force mixer (see Methods and Ref. ${ }^{[27]}$ for details). A bare film of the purified material shows the characteristic absorption and photoluminescence (PL) spectrum of (6,5) SWCNTs with an excitonic peak at $1010 \mathrm{~nm}$ (Figure 1a). As different selection procedures can yield different types of SWCNTs, ${ }^{[28]}$ we furthermore verified the purity of our samples by recording a 2D PL emission-excitation map (Supporting Information, Figure S1a). This confirmed exclusive emission from $(6,5)$ SWCNTs with no indication of further emitting SWCNTs. A topography image of a spincoated network reveals a high density and planar orientation of the nanotubes (Figure S1b).

The multi-layer bottom-emitting stack architecture of the SWCNT OLEDs used in this study is schematically illustrated in Figure 1b. The PEDOT:PSS hole transport layer (HTL) and the SWCNT emissive layer (EML) were deposited from solution while the low molecular weight hole blocking layer (HBL), the n-doped electron transport layer (ETL) and the cathode were deposited by thermal evaporation under high vacuum (see Methods). The thickness of the ETL was optimized for maximum outcoupling efficiency using a transfer-matrix based simulation algorithm. ${ }^{[29]}$ Due the long emission wavelength compared to conventional OLEDs, this resulted in a relatively thick ETL $(130 \mathrm{~nm})$. n-doping was applied to realize this thickness without causing a significant increase in operating voltage. ${ }^{[30]}$ Considering the simplified 


\section{WILEY-VCH}

energy level scheme of the OLED stack in Figure 1c, we expect that charges and excitons will be very efficiently trapped on the $(6,5) \operatorname{SWCNTs}\left(E_{\mathrm{HOMO}}=-5.08 \mathrm{eV}, E_{\mathrm{LUMO}}=-4.01 \mathrm{eV}\right.$ LUMO $)^{[31]}$. The selection method by which our monochiral SWCNTs are enriched leads to a single insulating polymer layer (PFO-BPy) around each nanotube through which charges can readily tunnel. Within the SWCNT network both electrons and holes are highly mobile, even across nanotube-nanotube junctions. ${ }^{[18,32]}$

We fabricated a series of OLEDs with SWCNT-layers of three different thicknesses (5, 16 and $39 \mathrm{~nm}$ ). For all thicknesses, OLEDs showed diode-like J-V characteristics and reached high current densities (180-320 $\mathrm{mA} \mathrm{cm}^{-2}$ at $\left.5 \mathrm{~V}\right)$ as expected from the high conductivity of the SWCNTs and the use of doped charge transport layers (Figure 2a). ${ }^{[33]}$ The intensity of EL, as recorded by a calibrated Ge photodiode, is shown in Figure $2 b$. The highest optical output power and lowest turn-on voltage were measured for the OLED with the $39 \mathrm{~nm}$ thick SWCNT layer. This device reaches $900 \mu \mathrm{W} \mathrm{cm}^{-2}$ at $300 \mathrm{~mA} \mathrm{~cm}^{-2}$ and turns on at $2.1 \mathrm{~V}$. Figure $2 \mathrm{c}$ shows the corresponding external quantum efficiency (EQE) as a function of current density. We observe a maximum EQE of $0.014 \%$ for the device with $39 \mathrm{~nm}$ thick SWCNT layer. The EQE is mainly limited by the PL quantum yield (PLQY) of the used SWCNT layer, which was $0.11 \%$. The different loss channels in the OLED are discussed in more detail later.

The EL spectra of the SWCNT OLEDs across the visible and nIR are given in Figure 2d. For OLEDs with a 39 nm-thick nanotube layer, we observed two emission peaks in the nIR and no detectable emission in the visible range. The emission peak at $1010 \mathrm{~nm}$ is associated with excitonic emission from $(6,5)$ SWCNTs and shows a narrow spectral width (FWHM, $41 \mathrm{~nm}$ ), consistent with PL measurements. Interestingly, an additional peak at $1177 \mathrm{~nm}$ with a FWHM of $58 \mathrm{~nm}$ was detected, which did not appear in the PL measurements (c.f. Figure 1b). Both, the peak position and the width of this peak suggest that it originates from the emission of trions, i.e. a charged excited state comprising one electron and two holes or vice versa. ${ }^{[34]}$ In our OLEDs, we most likely observe positively charged trions owing to 


\section{WILEY-VCH}

residual p-doping of the SWCNTs (due to remaining oxygen) as well as hole accumulation at the SWCNT/BAlq interface (due to the low conductivity of of BAlq compared to the other layers in the stack).This interpretation is also supported by the increase in the intensity of this peak relative to the exciton peak for devices with thinner SWCNT layers. For these devices the local charge carrier density in the SWCNT layer is expected to be larger (for the same current density), and thus the probability of trion generation increases. Additionally, however, devices with thin SWCNT layers show substantial emission in the visible part of the spectrum with two peaks at 430 and $570 \mathrm{~nm}$. We attribute these peaks to charge recombination in the adjacent blocking/transport layers and exciplex formation at the interfaces. An EML thickness of $>30 \mathrm{~nm}$ is thus desirable to generate pure nIR light at $>1000 \mathrm{~nm}$.

Trionic emission generally occurs due to excess charges in the emitter material and therefore typically increases with current density. This trend was indeed observed in our devices (Figure 3a), which further corroborates that emission is from a trion state rather than from a low-energy excitonic defect or trap state. (Any low-energy excitonic traps would be saturated at higher current densities and thus the relative emission intensity from these would decrease with respect to the main exciton peak.) Similar trion emission was previously observed from SWCNT based light-emitting field effect transistors (LEFETs) and its presence was explained by the high current densities in LEFETs (up to tens of $\mathrm{A} \mathrm{cm}^{-2}$ ). ${ }^{[34]}$ Surprisingly, however, the trion emission is even more pronounced in our OLEDs despite the generally lower current density $\left(<0.4 \mathrm{~A} \mathrm{~cm}^{-2}\right)$. We thus hypothesize that trion emission in our OLEDs is due to local hot spots of high charge density. A microscopic investigation of the EL across the pixel indeed showed spots with brighter emission than the surrounding (Figure $3 \mathrm{~b}$ and Figure S3). Note that the small part of the pixel shown in Figure $3 b$ is not representative for the entire pixel (c.f., Supporting Information Figure S3). As the EL intensity is to first approximation proportional to the local current density, this observation implies the presence of small regions of elevated current. These hot spots are also observed in co-located PL 


\section{WILEY-VCH}

images (Figure S3); we thus assume that they are associated with bundles of SWCNTs. Aggregates of nanotubes provide an increased density of states. In addition, they may enhance charge injection as well as charge transport through the SWCNT layer and thus carry more current than the surrounding parts. ${ }^{[35]}$ Using hyperspectral imaging, we found that the emission spectrum in the background area is dominated by exciton emission while the emission spectrum at the hot spot is characteristic of trion emission (Figure 3c).

Next, we investigated the orientation of the emissive dipoles in the EML. Since light emitted from horizontally oriented dipoles can be extracted from an OLED more efficiently than light from vertically oriented dipoles, much recent work in the OLED community has focused on preferentially aligning the emissive dipoles in the EML horizontally, i.e. parallel to the substrate. ${ }^{[36-38]}$ The extremely high aspect ratio of the carbon nanotubes (average length $1.8 \mu \mathrm{m}$, diameter $0.747 \mathrm{~nm}$ ) should lead to strong alignment of the emission dipoles parallel to the substrate. To investigate if this is indeed the case, we performed angle-resolved emission measurements as described in Refs. ${ }^{[39,40]}$. Figure 4a shows the angular resolved transverse magnetic polarized emission spectrum of an OLED with $39 \mathrm{~nm}$ thick EML. These data were fitted to transfer matrix simulations of the emission, using the anisotropy factor $a$, i.e. the fraction of vertically oriented dipoles in the film, as the fit parameter. The best agreement between the experimental spectra and the transfer matrix simulations was obtained for $a=0.05$ (Figure 4b). A simulation that assumes isotropic orientation $(a=1 / 3)$ clearly deviates from the experimental data (Figure 4c), whereas a simulation assuming perfect horizontal orientation of the transition dipole moments $(a=0)$ resembles the experimental data nearly as well as the best fit (Figure 4d). The obtained anisotropy factor of 0.05 corresponds to an average inclination angle of $12.9^{\circ}$ with respect to the surface. Since the transition dipole moments of both excitons and trions in SWCNTs are parallel to the tube axis, this result is in agreement with the flat orientation of the SWCNTs. ${ }^{[34]}$ 


\section{WILEY-VCH}

Based on an anisotropy factor of 0.05 and using the experimentally measured EL spectrum of our OLEDs in forward direction, our transfer matrix calculation predicts that the outcoupling efficiency for our devices is $49 \%$, which is $60 \%$ higher than for isotropically oriented emissive dipoles. The predominant remaining optical loss channels are thus coupling to substrate modes, wave-guiding in the organic layers and excitation of lossy surface plasmon modes. Considering a PLQY of $0.11 \%$, an outcoupling efficiency of $49 \%$ and a singlet fraction of 0.25 , and assuming perfect charge balance, the maximum achievable EQE is estimated to $0.014 \%$. The best devices in this study approach this value at low current density (see Figure 2c). While the exchange energy for triplets in $(6,5)$ SWNT is about $120 \mathrm{meV}$, the allowed conversion of dark triplets into bright trions may provide an additional route toward radiative relaxation. ${ }^{[41]}$ For all devices, the EQE rolls off very significantly at higher brightness levels. Besides potential annihilation processes, ${ }^{[42]}$ Auger quenching ${ }^{[34]}$ is likely responsible for this strong efficiency roll-off.

In conclusion, we demonstrated the first OLEDs that employ SWCNTs as emitters to generate electroluminescence exclusively in the nIR (> $1000 \mathrm{~nm})$. Doping facilitated the realization of the thick ETL required for efficient light extraction at nIR wavelengths. Although the EQE of these first devices is limited to $0.014 \%$, SWCNT OLEDs offer several intriguing advantages: SWCNTs are free of heavy metals and could be fabricated on flexible substrates, for example, leading to wearable devices for applications in skin treatment. Furthermore, the robustness of SWCNTs in terms of photostability and thermal stability ${ }^{[18]}$ will be beneficial for long-term stability. In the future, we expect that the EQE can be increased dramatically by using chemically modified SWCNTs, which were shown to offer much higher brightness by harvesting dark excitons. ${ }^{[43,44]}$ The use of advanced deposition methods, such as aerosol jet printing ${ }^{[45]}$, will likely lead to more homogeneous SWCNT films and thus more evenly distributed EL and reduced trion emission, thus further improving spectral purity. In addition, by using SWCNTs with different diameters and chirality ${ }^{[28]}$ or 


\section{WILEY-VCH}

through chemical modification, ${ }^{[43]}$ OLEDs with emission across the entire nIR could be realized.

\section{Experimental Section}

SWCNT selection: Selective polymer-wrapping was used to prepare monochiral dispersions of (6,5) SWCNTs. ${ }^{[27]}$ First, 0.5 g/L PFO-BPy (poly[(9,9-dioctylfluorenyl-2,7-diyl)-alt-co-(6,6'$\{2,2$ '-bipyridine $\})]$, American Dye Source, $\mathrm{M}_{\mathrm{W}}=34 \mathrm{~kg} / \mathrm{mol}$ ) was dissolved in $140 \mathrm{~mL}$ toluene and then $0.38 \mathrm{~g} / \mathrm{L}$ CoMoCAT® raw material (773735, Lot \#14J017A1, Sigma Aldrich) was added. Next, high-speed shear force mixing (Silverson L5M-A) was applied at maximum speed $(10,230 \mathrm{rpm})$ for $72 \mathrm{~h}$ at constant temperature of $20^{\circ} \mathrm{C}$. After dispersion, the mixture was centrifuged at 50,000 g for $60 \mathrm{~min}$ (Beckman Coulter Avanti J26XP centrifuge), with an intermediate supernatant extraction after $30 \mathrm{~min}$. The high purity $(6,5) \mathrm{SWCNTs}$ dispersion in the supernatant were further enriched by pelleting via ultracentrifugation at $284,600 \mathrm{~g}$. Different amount of these pellets were then redispersed in toluene for spin-coating. Device Fabrication: A $40 \mathrm{~nm}$ thick layer of poly(3,4-ethylenedioxythiophene) polystyrene sulfonate (PEDOT:PSS) was spin-coated onto a $1.1 \mathrm{~mm}$ thick glass substrate with a $90 \mathrm{~nm}$ thick pre-patterned indium tin oxide (ITO) anode. After annealing at $120{ }^{\circ} \mathrm{C}$ for $20 \mathrm{~min}$, the SWCNT layer was spin-coated at $2000 \mathrm{rpm}$ and baked for $30 \mathrm{~min}$ at $120{ }^{\circ} \mathrm{C}$ in a dry nitrogen environment. By varying the SWCNT concentration, 5, 16 and $39 \mathrm{~nm}$ thick EML layers were obtained. Subsequently, samples were transferred to a high vacuum thermal evaporation system operating at a base pressure of $2 \times 10^{-7} \mathrm{mbar}$ (Angstrom EvoVac). $10 \mathrm{~nm}$ bis(2-methyl8-quinolinolate)-4(phenylphenolato)-aluminium(III) (BAlq) and $130 \mathrm{~nm}$ Cs-doped 4,7diphenyl-1,10-phenanthroline (BPhen) were evaporated as HBL and ETL, respectively. The devices were completed with a $100 \mathrm{~nm}$ thick aluminum electrode and subsequently encapsulated under nitrogen atmosphere with a glass lid and getter. The active device area was $16.1 \mathrm{~mm}^{2}$. 


\section{WILEY-VCH}

Device Characterization: Current-voltage-brightness characteristics were recorded with a source-measurement unit (Keithley, SMU2400) and a calibrated Ge photodetector (Thorlabs, PDA50B-EC) with a dichroic $900 \mathrm{~nm}$ long-pass filter. The EQE was calculated from the forward emission intensity and spectrum, assuming a Lambertian emission pattern.

To measure EL spectra, OLEDs were placed $15 \mathrm{~cm}$ away from the entrance of an optical fiber. For nIR measurements, the EL collected by the fiber was coupled into an Acton SpectraPro SP2358 spectrometer (grating 150 lines $/ \mathrm{mm}$ ) and a liquid-nitrogen-cooled InGaAs line camera (Princeton Instruments OMAV). A fiber spectrometer with silicon CCD detector (Ocean Optics USB4000-UV-VIS) was used to record EL spectra in the visible. The two types of spectra were stitched by overlaying them in the 850 to $1050 \mathrm{~nm}$ region as shown in Fig. S2. For angular spectroscopy, an additional polarization filter was used to transmit only the transverse magnetic polarization and the OLED was mounted on a rotation stage. EL spectra were taken in $5^{\circ}$ steps. In order to ensure there was no substantial degradation of the OLEDs during the measurement, the spectrum at $0^{\circ}$ was re-measured after the angle-sweep. The peak intensity was found to be within $95 \%$ of the original intensity.

Micrographs and hyperspectral images were recorded with a home-build microscope setup equipped with a dichroic long-pass filter (cut-off wavelength, 850nm), a spectrograph (Princeton Instruments IsoPlane) and a thermoelectrically cooled 640×512 InGaAs array camera (Princeton Instruments, NIRvana:640ST).

\section{Supporting Information}

Supporting Information is available from the Wiley Online Library or from the author.

\section{Acknowledgements}

This research was financially supported by the Volkswagen Foundation (93404), the European Research Council under the European Union's Seventh Framework Programme 


\section{WILEY-VCH}

(FP/2007-2013)/ERC Grant Agreement No. 306298 (EN-LUMINATE) and under the

European Union's Horizon 2020 Framework Programme (FP/2014-2020)/ERC Grant Agreement No. 640012 (ABLASE), and by EPSRC (EP/R010595/1). C.M. acknowledges funding by the European Commission through a Marie Skłodowska Curie Individual Fellowship (703387). J.Z. thanks the Alfried Krupp von Bohlen und Halbach-Stiftung via the “Alfried Krupp Förderpreis für junge Hochschullehrer” for general support.

Received: ((will be filled in by the editorial staff))

Revised: ((will be filled in by the editorial staff)) Published online: ((will be filled in by the editorial staff))

\section{References}

[1] K. Tuong Ly, R.-W. Chen-Cheng, H.-W. Lin, Y.-J. Shiau, S.-H. Liu, P.-T. Chou, C.-S. Tsao, Y.-C. Huang, Y. Chi, Nat. Photonics 2017, 11, 63.

[2] A. Zampetti, A. Minotto, B. M. Squeo, V. G. Gregoriou, S. Allard, U. Scherf, C. L. Chochos, F. Cacialli, Sci. Rep. 2017, 7, 1611.

[3] S. Kim, H. J. Kwon, S. Lee, H. Shim, Y. Chun, W. Choi, J. Kwack, D. Han, M. Song, S. Mohammadi, I. Kee, S. Y. Lee, Adv. Mater. 2011, 23, 3511.

[4] M. C. Gather, A. Köhnen, K. Meerholz, Adv. Mater. 2011, 23, 233.

[5] Z. Y. Wang, in Near-Infrared Org. Mater. Emerg. Appl., CRC Press, 2013, pp. 115170.

[6] R. Nagata, H. Nakanotani, C. Adachi, Adv. Mater. 2017, 29, 1604265.

[7] A. Shahalizad, A. D’Aléo, C. Andraud, M. H. Sazzad, D.-H. Kim, Y. Tsuchiya, J.-C. Ribierre, J.-M. Nunzi, C. Adachi, Org. Electron. 2017, 44, 50.

[8] F. Yan, W. Li, B. Chu, H. Liu, G. Zhang, Z. Su, J. Zhu, L. Han, T. Li, Y. Chen, C. H. Cheng, Z. Q. Fan, G. T. Du, Org. Electron. 2009, 10, 1408.

[9] G. Qian, Z. Zhong, M. Luo, D. Yu, Z. Zhang, Z. Y. Wang, D. Ma, Adv. Mater. 2009, $21,111$. 


\section{WILEY-VCH}

[10] G. Tregnago, T. T. Steckler, O. Fenwick, M. R. Andersson, F. Cacialli, J. Mater. Chem. C 2015, 3, 2792.

[11] N. Tessler, V. Medvedev, M. Kazes, S. Kan, U. Banin, Science 2002, 295, 1506.

[12] X. Gong, Z. Yang, G. Walters, R. Comin, Z. Ning, E. Beauregard, V. Adinolfi, O. Voznyy, E. H. Sargent, Nat. Photonics 2016, 10, 253.

[13] A. Nish, J. Y. Hwang, J. Doig, R. J. Nicholas, Nat. Nanotechnol. 2007, 2, 640.

[14] X. He, N. F. Hartmann, X. Ma, Y. Kim, R. Ihly, J. L. Blackburn, W. Gao, J. Kono, Y. Yomogida, A. Hirano, T. Tanaka, H. Kataura, H. Htoon, S. K. Doorn, Nat. Photonics 2017, 11,577 .

[15] A. T. Mallajosyula, W. Nie, G. Gupta, J. L. Blackburn, S. K. Doorn, A. D. Mohite, ACS Nano 2016, 10, 10808.

[16] M. Pfohl, K. Glaser, A. Graf, A. Mertens, D. D. Tune, T. Puerckhauer, A. Alam, L. Wei, Y. Chen, J. Zaumseil, A. Colsmann, R. Krupke, B. S. Flavel, Adv. Energy Mater. 2016, 6, 1600890.

[17] S. Khasminskaya, F. Pyatkov, K. Słowik, S. Ferrari, O. Kahl, V. Kovalyuk, P. Rath, A. Vetter, F. Hennrich, M. M. Kappes, G. Gol’tsman, A. Korneev, C. Rockstuhl, R. Krupke, W. H. P. Pernice, Nat. Photonics 2016, 10, 727.

[18] A. Graf, M. Held, Y. Zakharko, L. Tropf, M. C. Gather, J. Zaumseil, Nat. Mater. 2017, $16,911$.

[19] L. Li, J. Liang, S.-Y. Chou, X. Zhu, X. Niu, ZhibinYu, Q. Pei, Sci. Rep. 2014, 4, 4307.

[20] P. Freitag, A. A. Zakhidov, B. Luessem, A. A. Zakhidov, K. Leo, J. Appl. Phys. 2012, 112, 114505.

[21] C. M. Aguirre, S. Auvray, S. Pigeon, R. Izquierdo, P. Desjardins, R. Martel, Appl. Phys. Lett. 2006, 88, 18.

[22] D. Zhang, K. Ryu, X. Liu, E. Polikarpov, J. Ly, M. E. Tompson, C. Zhou, Nano Lett. 2006, 6, 1880 . 


\section{WILEY-VCH}

[23] R. C. Tenent, T. M. Barnes, J. D. Bergeson, A. J. Ferguson, B. To, L. M. Gedvilas, M. J. Heben, J. L. Blackburn, Adv. Mater. 2009, 21, 3210.

[24] H. S. Woo, R. Czerw, S. Webster, D. L. Carroll, J. Ballato, A. E. Strevens, D. O’Brien, W. J. Blau, Appl. Phys. Lett. 2000, 77, 1393.

[25] P. Fournet, J. N. Coleman, B. Lahr, A. Drury, W. J. Blau, D. F. O’Brien, H.-H. Hörhold, J. Appl. Phys. 2001, 90, 969.

[26] Y.-G. Ha, E.-A. You, B.-J. Kim, J.-H. Choi, Synth. Met. 2005, 153, 205.

[27] A. Graf, Y. Zakharko, S. P. Schieß1, C. Backes, M. Pfohl, B. S. Flavel, J. Zaumseil, Carbon N. Y. 2016, 105, 593.

[28] H. Liu, D. Nishide, T. Tanaka, H. Kataura, Nat. Commun. 2011, 2, 309.

[29] M. Furno, R. Meerheim, S. Hofmann, B. Lüssem, K. Leo, Phys. Rev. B 2012, 85, 115205.

[30] B. Lüssem, M. Riede, K. Leo, Phys. status solidi 2013, 210, 9.

[31] Y. Tanaka, Y. Hirana, Y. Niidome, K. Kato, S. Saito, N. Nakashima, Angew. Chemie Int. Ed. 2009, 48, 7655 .

[32] S. P. Schieß1, N. Fröhlich, M. Held, F. Gannott, M. Schweiger, M. Forster, U. Scherf, J. Zaumseil, ACS Appl. Mater. Interfaces 2015, 7, 682.

[33] C. Murawski, C. Fuchs, S. Hofmann, K. Leo, M. C. Gather, Appl. Phys. Lett. 2014, 105, 113303.

[34] F. Jakubka, S. B. Grimm, Y. Zakharko, F. Gannott, J. Zaumseil, ACS Nano 2014, 8, 8477.

[35] A. Malhofer, M. Rother, Y. Zakharko, A. Graf, S. P. Schieß1, J. Zaumseil, Org. Electron. 2017, 45, 151.

[36] M. Flämmich, M. C. Gather, N. Danz, D. Michaelis, A. H. Bräuer, K. Meerholz, A. Tünnermann, Org. Electron. 2010, 11, 1039.

[37] K.-H. Kim, S. Lee, C.-K. Moon, S.-Y. Kim, Y.-S. Park, J.-H. Lee, J. Woo Lee, J. Huh, 


\section{WILEY-VCH}

Y. You, J.-J. Kim, Nat. Commun. 2014, 5, 4769.

[38] W. Brütting, J. Frischeisen, T. D. Schmidt, B. J. Scholz, C. Mayr, Phys. Status Solidi 2013, $210,44$.

[39] P. Liehm, C. Murawski, M. Furno, B. Lüssem, K. Leo, M. C. Gather, Appl. Phys. Lett. 2012, 101, 253304.

[40] A. Graf, P. Liehm, C. Murawski, S. Hofmann, K. Leo, M. C. Gather, J. Mater. Chem. C 2014, 2, 10298.

[41] K. Watanabe, K. Asano, Phys. Rev. B 2012, 85, 35416.

[42] C. Murawski, K. Leo, M. C. Gather, Adv. Mater. 2013, 25, 6801.

[43] Y. Piao, B. Meany, L. R. Powell, N. Valley, H. Kwon, G. C. Schatz, Y. Wang, Nat. Chem. 2013, 5, 840 .

[44] N. F. Hartmann, R. Pramanik, A.-M. Dowgiallo, R. Ihly, J. L. Blackburn, S. K. Doorn, ACS Nano 2016, 10, 11449.

[45] M. Rother, M. Brohmann, S. Yang, S. B. Grimm, S. P. Schieß1, A. Graf, J. Zaumseil, Adv. Electron. Mater. 2017, 3, 1700080. 


\section{WILEY-VCH}

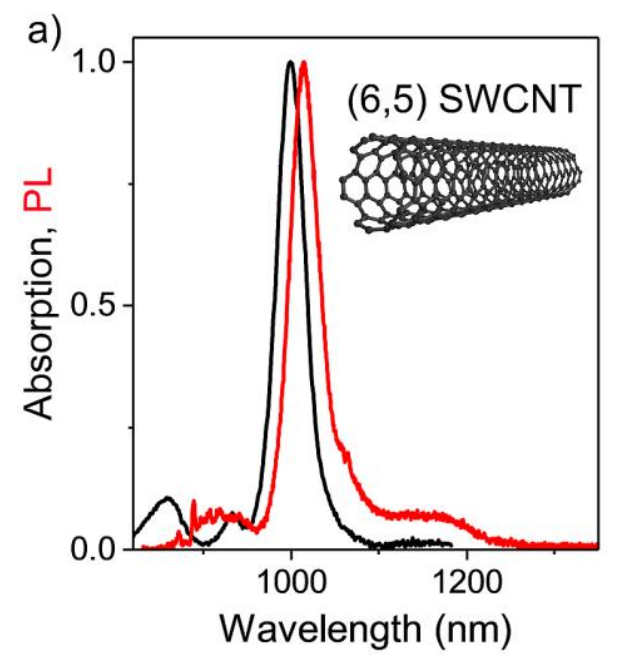

b)

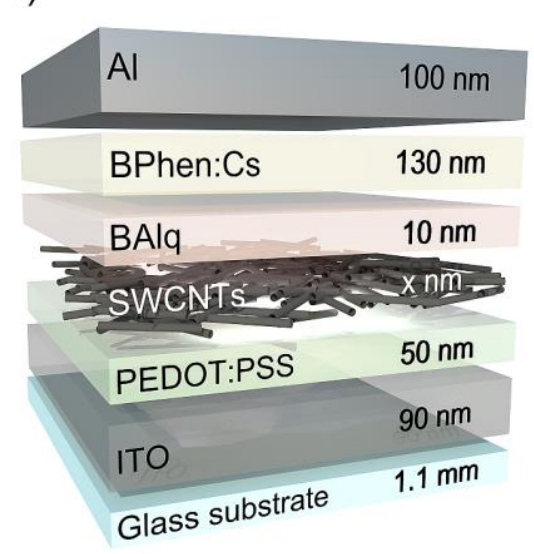

c)

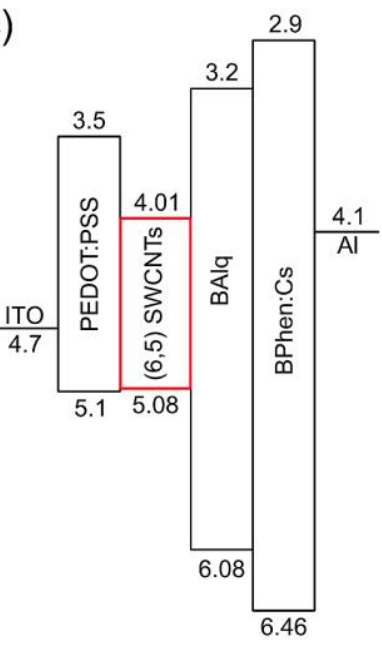

Figure 1. a) Absorption and photoluminescence (PL) spectra of the $(6,5)$ SWCNTs used as emitter material. The inset shows the molecular structure of a $(6,5)$ SWCNT. b) Schematic illustration and c) proposed energy level diagram for the OLED stack used in this work. 


\section{WILEY-VCH}
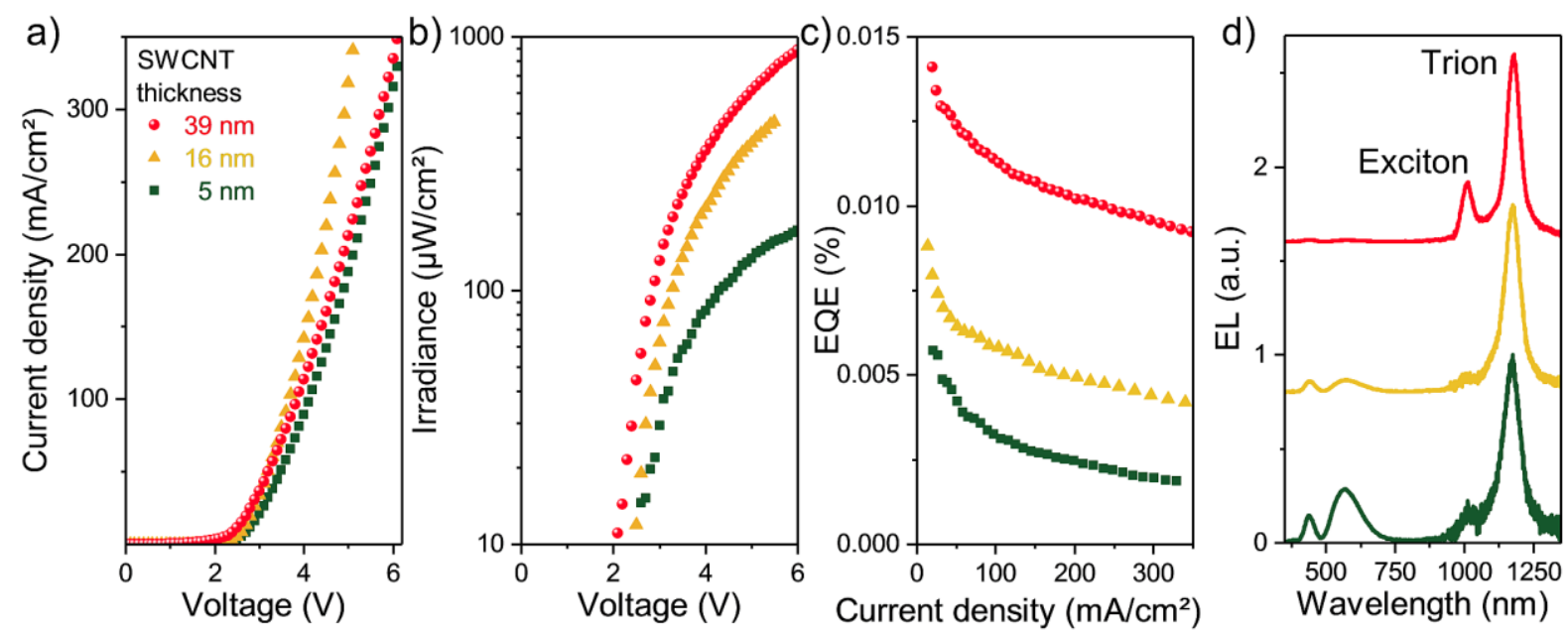

Figure 2. Characteristics of OLEDs with SWCNT emissive layers of different thickness. a) Current density and b) nIR irradiance as function of bias voltage. From red to green the emissive layer thickness decreases. c) External quantum efficiency versus current density. d) Electroluminescence spectra of the OLEDs emitted in forward direction, at a current density of $62 \mathrm{~mA} \mathrm{~cm}^{-2}$. 


\section{WILEY-VCH}
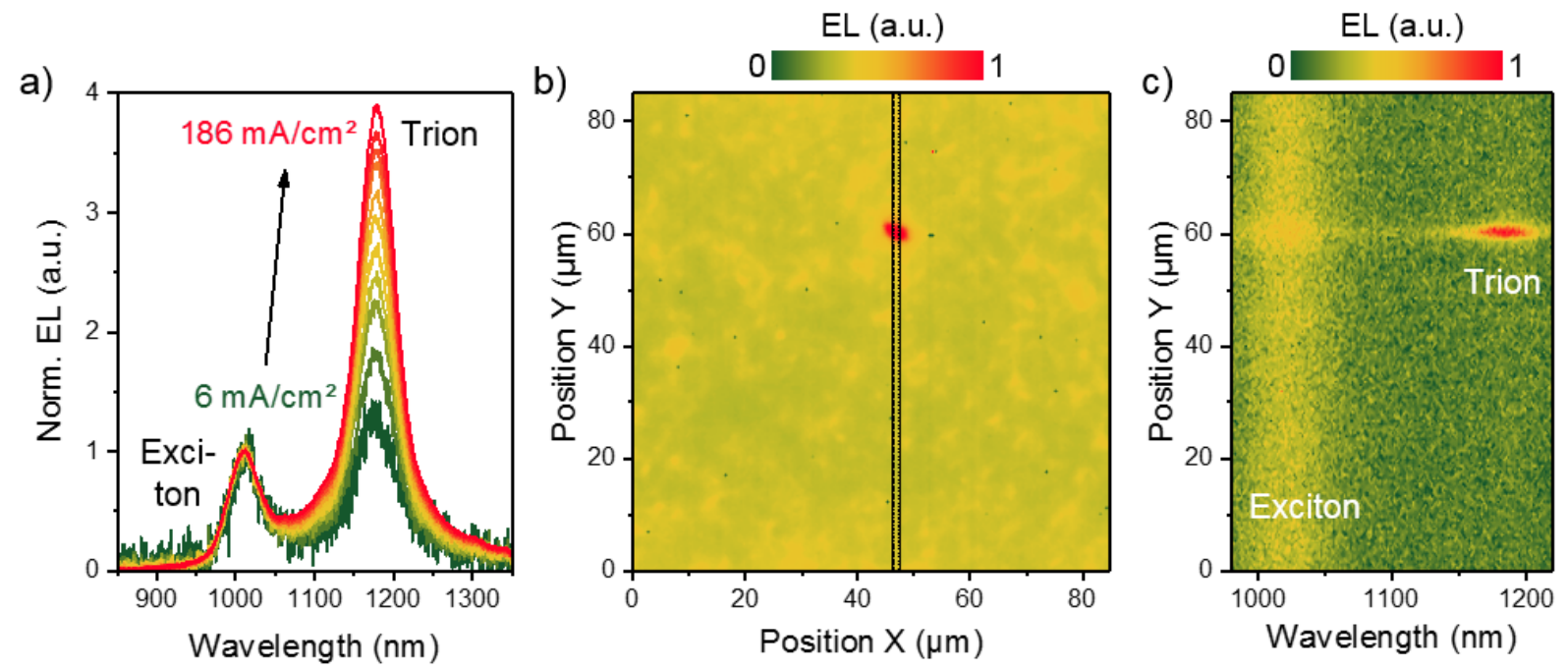

Figure 3. Characteristics of exciton and trion emission. a) Current-dependent EL spectrum (normalized to the exciton emission peak) for an OLED with a $39 \mathrm{~nm}$ thick SWCNT layer. b) Real-space micrograph of the EL signal from the same OLED at $62 \mathrm{~mA} \mathrm{~cm}{ }^{-2}$ revealing the presence of an emission hot-spot. c) Hyperspectral image along the line marked in b). Excitonic emission is observed along the entire line while bright trion emission dominates in the region of the hot-spot. 


\section{WILEY-VCH}
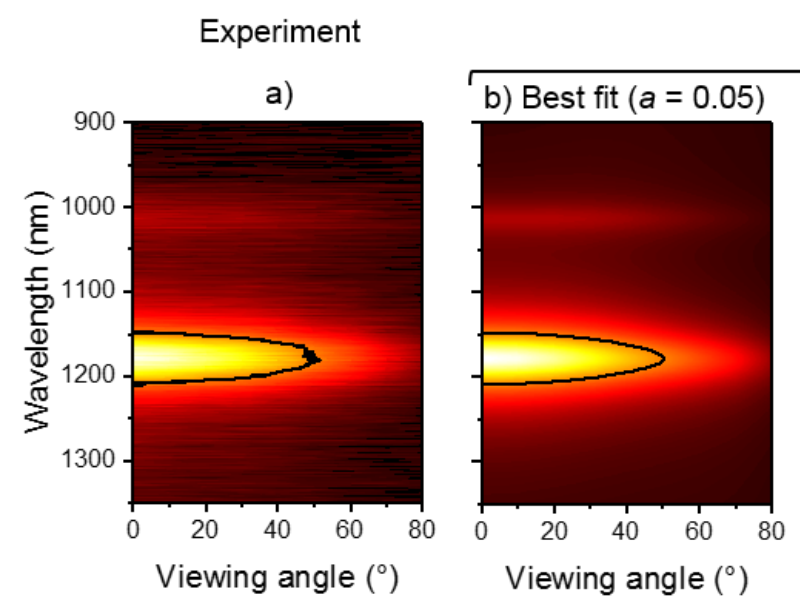

Simulation

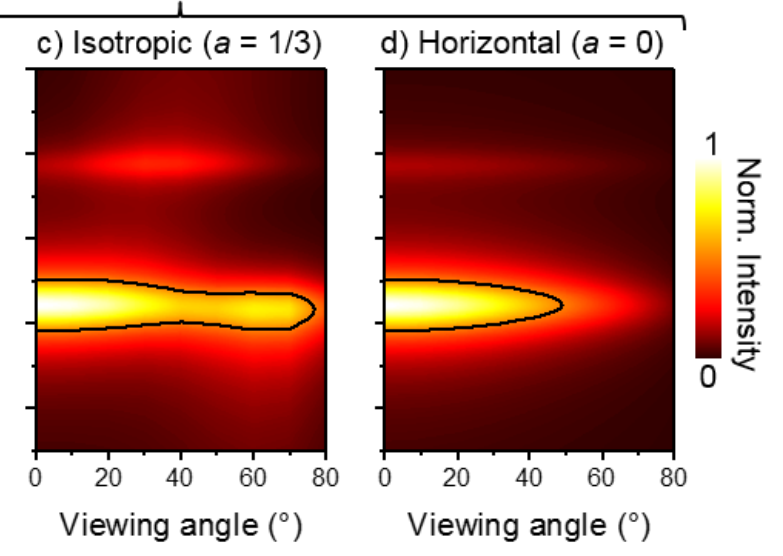

Figure 4. Orientation of SWCNT emissive dipoles. a) Angle-resolved TM-polarized EL spectrum of an OLED with $39 \mathrm{~nm}$ thick SWCNT layer, measured at $124 \mathrm{~mA} \mathrm{~cm}^{-2}$ current density. b) Best fit to the experimental data is obtained for an anisotropy factor of 0.05 , corresponding to an average inclination angle of emissive dipoles with respect to the substrate of $12.9^{\circ}$. c) and d) Simulated emission intensity for isotropic and exclusively horizontal transition dipole orientation, respectively. 


\section{WILEY-VCH}

The first organic light-emitting diode with semiconducting single-walled carbon nanotubes as the emitter is demonstrated. Excitonic and trionic emission of the employed $(6,5)$ nanotubes gives narrow band electroluminescence between $1000 \mathrm{~nm}$ and $1200 \mathrm{~nm}$. The emissive dipole orientation shows strong horizontal alignment of the nanotubes leading to an exceptionally high outcoupling efficiency of $49 \%$.

Keyword: OLED, single-walled carbon nanotube, near infrared, trion, emitter orientation

Arko Graf, Caroline Murawski, Yuriy Zakharko, Jana Zaumseil* and Malte C. Gather*

Infrared organic light-emitting diodes with carbon nanotube emitters

ToC figure

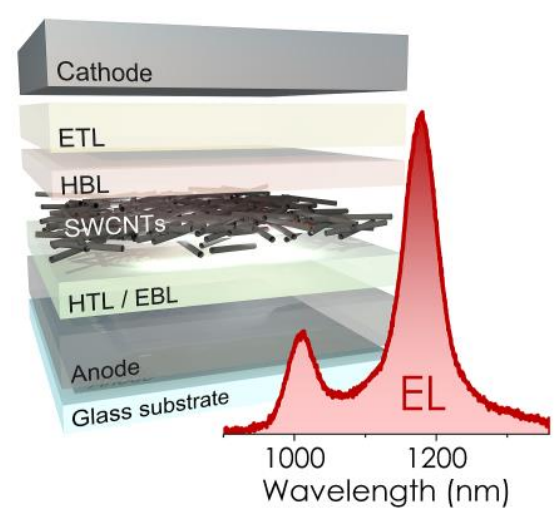




\section{WILEY-VCH}

Copyright WILEY-VCH Verlag GmbH \& Co. KGaA, 69469 Weinheim, Germany, 2016.

\section{Supporting Information}

Infrared organic light-emitting diodes with carbon nanotube emitters

Arko Graf, Caroline Murawski, Yuriy Zakharko, Jana Zaumseil* and Malte C. Gather*

A. Graf, Dr. Y. Zakharko, Prof. J. Zaumseil

Institute for Physical Chemistry, Universität Heidelberg, D-69120 Heidelberg, Germany

E-mail: zaumseil@uni-heidelberg.de

A. Graf, Dr. C. Murawski, Prof. M. C. Gather

Organic Semiconductor Centre, SUPA, School of Physics and Astronomy,

University of St Andrews, St Andrews KY16 9SS, United Kingdom

E-mail: $\underline{\operatorname{mcg} 6 @ \text { st-andrews.ac.uk }}$ 


\section{WILEY-VCH}

a)

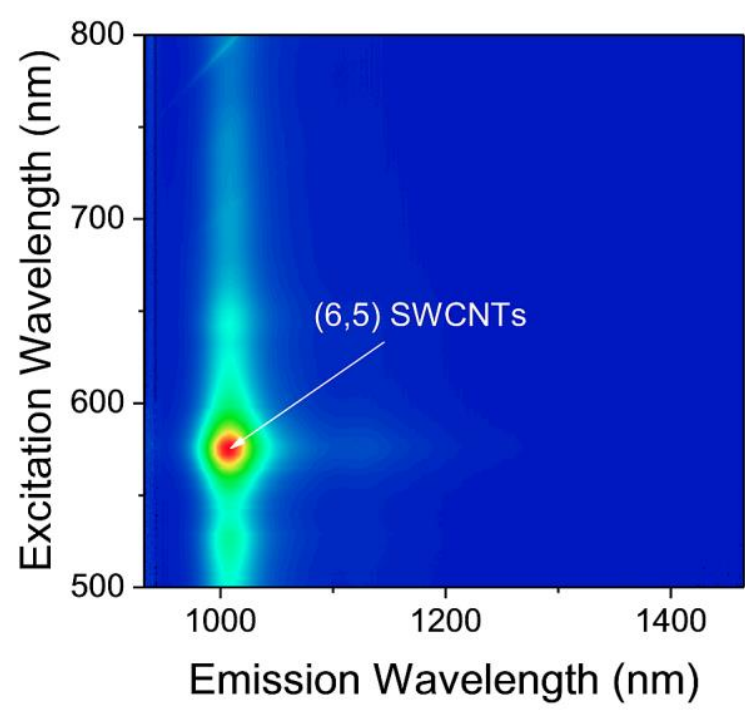

b)

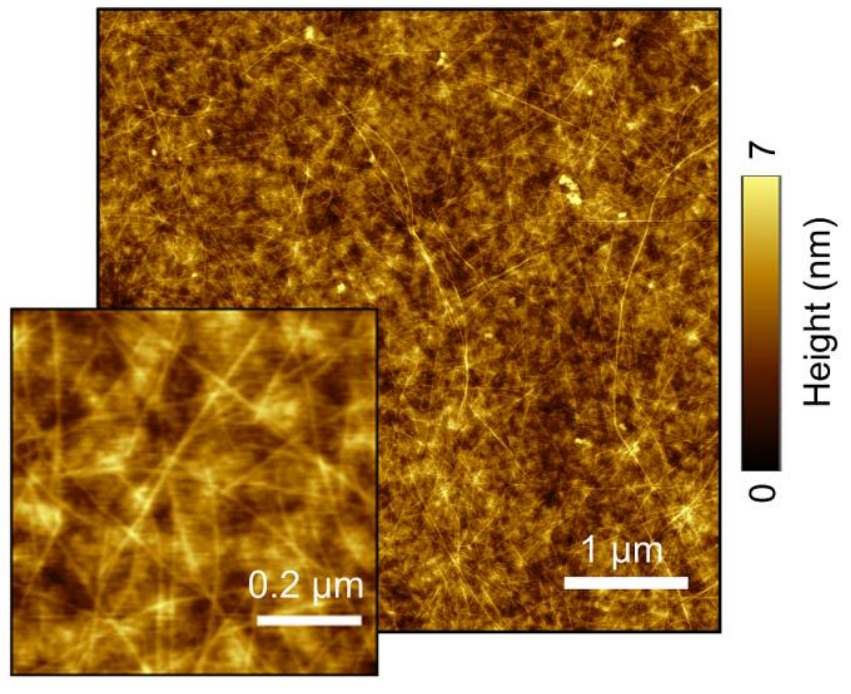

Figure S1. a) PL-excitation map for a thin film of the $(6,5)$ SWCNTs used in this work. b) Atomic force microscope topography image of a thin film of $(6,5)$ SWCNTs, showing the nanotube network with strong horizontal orientation. 


\section{WILEY-VCH}

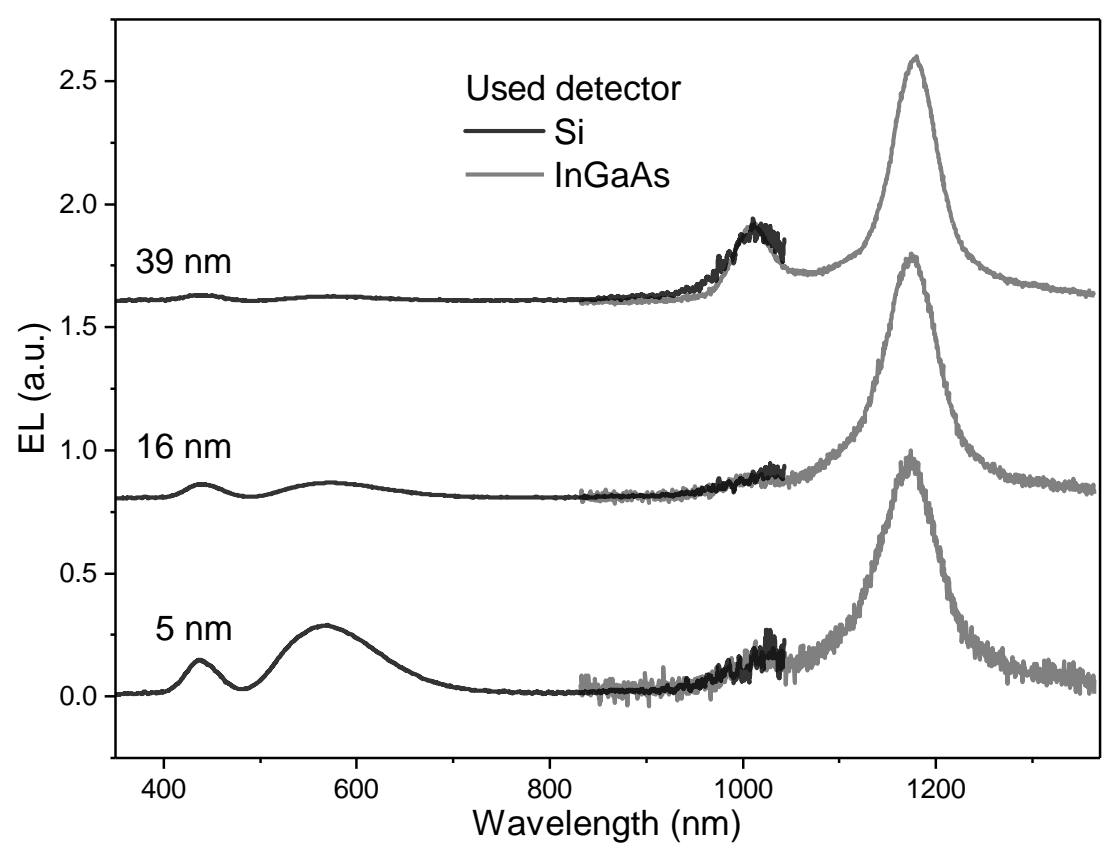

Figure S2. Emission spectra of the OLEDs produced in this work across the visible to near infrared region. Spectra are corrected for detection efficiency and stitched by normalizing them to each other in the region between $850 \mathrm{~nm}$ and $1050 \mathrm{~nm}$. 


\section{WILEY-VCH}

a)

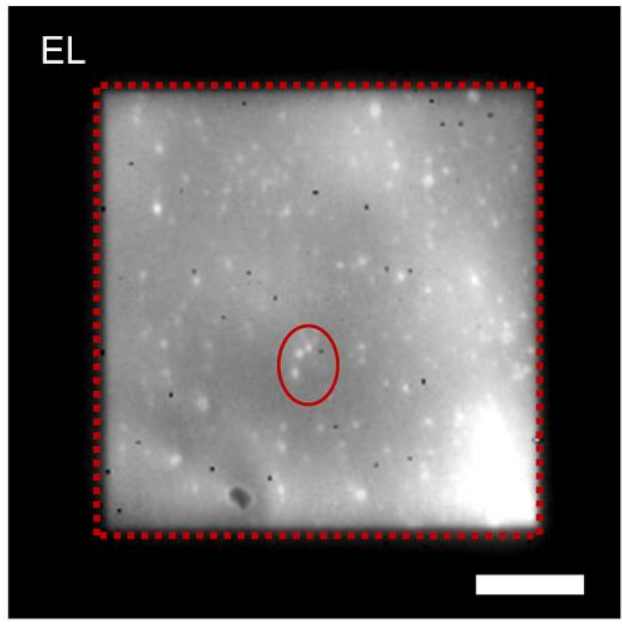

b)

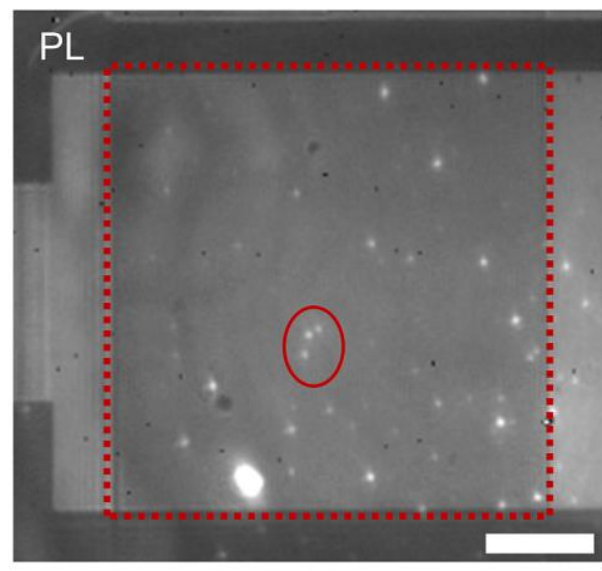

Figure S3. a) Image of the EL emitted by a single $\sim 4 \mathrm{~mm} \times 4 \mathrm{~mm}$ OLED pixel. b) Corresponding PL signal for homogeneous excitation. Scale bar: $1 \mathrm{~mm}$. 